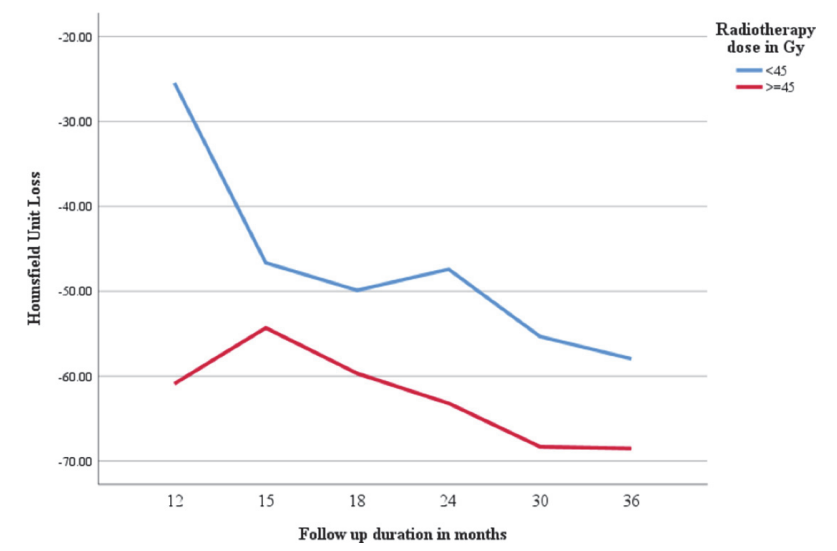

Abstract 389 Figure 1

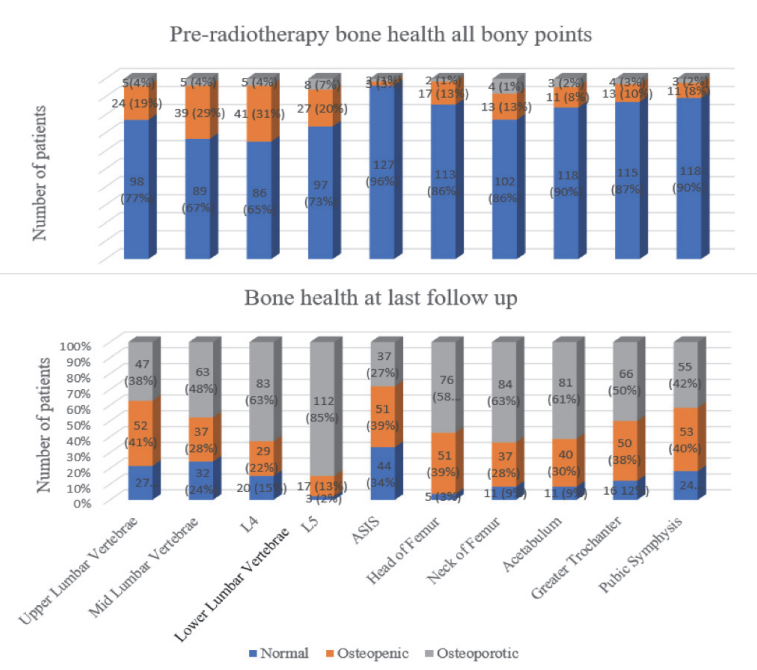

Abstract 389 Figure 2

and multivariate analysis was performed. Additionally, linear mixed model was used to report interaction of follow up duration and RT dose.

Result(s)* Overall 132 patients were included. The median RT doses was as follows: L1-L2: 1.2-2.1 Gy (1.1-2.4Gy), L4:11 Gy (7.5-17.8 Gy), L5: 47 Gy (42.6-49.3 Gy), Femur: 44-48 Gy (41-50 Gy), Acetabulum: 48 Gy (42-49 Gy), Greater Trochanter 26-30 Gy (17-35 Gy). The median HU loss was 33 HU for doses between 1-11 Gy, 45 HU for12-25 Gy and 60 HU for 26-50 Gy. Before RT, 96\% patients had normal bone health. At 24 months only $3 \%$ had normal bone health whereas $85 \%$ were osteoporotic $(p<0.001)$. Both RT dose $(\mathrm{p}<0.02)$ and time $(\mathrm{p}<0.001)$ predicted for BD loss whereas interaction of dose $x$ time was not significant $(p=0.56)$. No other patient and treatment related factors predicted for BD changes on univariate analysis. Multivariate analysis was not performed.

Conclusion* RT doses correlated with BD loss in cohort of patients undergoing postoperative pelvic RT. The results highlight the need for structured evaluation of bone density after pelvic RT.

\section{CLINICAL EVALUATION OF DNA METHYLATION AND HPV DNA TESTING IN URINE FOR CERVICAL INTRAEPITHELIAL NEOPLASIA AND CERVICAL CANCER DETECTION}

${ }^{1 ; 2} \mathrm{R}$ Van den Helder, ${ }^{1} \mathrm{RDM}$ Steenbergen, ${ }^{1} \mathrm{AP}$ Van Splunter, ${ }^{3} \mathrm{I}$ Martins, ${ }^{4} \mathrm{CH}$ Mom, ${ }^{4} \mathrm{MY}$ Tjiong, ${ }^{5} \mathrm{~F}$ Rosier-van Dunné, ${ }^{6} \mathrm{IAM}$ Van der Avoort, ${ }^{1} \mathrm{MCG}$ Bleeker, ${ }^{2} \mathrm{~N}$ Van Trommel. ${ }^{1}$ Amsterdam University Medical Center (UMC), Vrije Universiteit Amsterdam, Department of Pathology, Amsterdam UMC, Netherlands; ${ }^{2}$ Antoni van Leeuwenhoek/Netherlands Cancer Institute, Center of Gynecologic Oncology Amsterdam, Department of Gynecologic Oncology, Amsterdam, Netherlands; ${ }^{3}$ Amsterdam University Medical Center (UMC), Vrije Universiteit Amsterdam, Department of Epidemiology and Biostatistics, Amsterdam, Netherlands; ${ }^{4}$ Amsterdam University Medical Center (UMC), Amsterdam Medical Center, Department of Gynecologic Oncology, Amsterdam, Netherlands; ${ }^{5}$ Tergooi, Department of Obstetrics and Gynecology, Hilversum, Netherlands; ${ }^{6}$ Ikazia Hospital Rotterdam, Department of Obstetrics and Gynecology, Rotterdam, Netherlands

\subsection{6/ijgc-2021-ESGO.29}

Introduction/Background* Urine, as a liquid biopsy, can be obtained easily and noninvasively. Urine sampling might increase effectiveness of cervical cancer screening programs, by attracting women currently unreached by conventional screening methods (e.g. repetitive non-responders). An emerging biomarker for early cancer detection is DNA methylation. Altered DNA methylation is a common epigenetic event that occurs during the early stages of carcinogenesis, and has been linked to gene silencing of tumor suppressor genes. We aimed to determine the performance of high risk human papillomavirus (hrHPV) and host cell gene DNA methylation testing in urine for cervical cancer and high-grade cervical intraepithelial neoplasia (CIN2 and CIN3) detection. Paired cervicovaginal samples, used for conventional cervical cancer screening, were tested for comparison.

Methodology A total of 269 women were included in this study: 113 women diagnosed with cervical cancer (paired urine samples, cervicovaginal self-samples and cervical scrapes), 92 women diagnosed with a CIN2 or CIN3 lesion (paired urine samples and cervicovaginal self-samples) and 64 healthy female controls (urine samples). Samples were tested for five DNA methylation markers (ASCL1, GHSR, LHX8, SST, ZIC1) and hrHPV DNA. Methylation levels in urine were compared, performance was calculated based on AUCs and logistic regression, and a marker panel was obtained by multivariable logistic regression. Agreement within samples was determined using Cohen's kappa statistics and the Spearman correlation coefficients.

Result(s)* All markers in urine increased significantly with severity of disease, marker panel ASCL1/LHX8 resulted in an AUC of 0.84 for cervical cancer and CIN3 (CIN3+) detection, with a sensitivity of $77 \%$ and $86 \%$, at a predefined specificity of $80 \%$ and $70 \%$. In samples from women with cervical cancer, 83\% hrHPV-positivity and 94\% ASCL1/LHX8positivity was found in urine, $88 \%$ and $94 \%$ in self-samples, and $92 \%$ and $98 \%$ in cervical scrapes, respectively. Between paired samples from women with CIN2/3 and cervical cancer, a fair to strong correlation for methylation markers and a moderate to strong agreement for hrHPV DNA was found.

Conclusion* For women currently unreached by conventional screening methods, DNA methylation and hrHPV DNA testing in urine offers a promising solution to detect cervical cancer and high grade CIN lesions. 